\title{
Electrocardiographic QT Prolongation after Ablation of the Nodose Placode in the Chick Embryo: A Developmental Model of the Idiopathic Long QT Syndrome ${ }^{1}$
}

\author{
JAMES L. CHRISTIANSEN, HARRIET A. STADT, MICHAEL J. MULROY, AND \\ MARGARET L. KIRBY \\ Heart Development Group, Department of Anatomy, Medical College of Georgia, Augusta, Georgia 30912-2000
}

\begin{abstract}
Electrocardiographic abnormalities characteristic of the idiopathic long QT syndrome are thought to be caused by an imbalance of sympathetic activity in the heart. Recent evidence indicates that autonomic and sensory innervation density in the end-organ is modulated by reciprocal interactions. Ablation of one neuronal population allows reciprocal increases in growth of the remaining nerves. To test whether QT prolongation could be produced in chick embryos by altering sensory innervation to the heart, microcautery was used to ablate premigratory areas of the right nodose placode, a coalescence of cells in the embryonic ectoderm that generates neurons providing sensory innervation to the heart via the inferior ganglion of the vagus (nodose ganglion). After functional autonomic innervation was established, three-lead ECG were obtained in embryos with the right nodose placode ablated (experimental) and in sham-operated controls (sham) at incubation days 17-20 in a controlled temperature environment. Electrocardiograms were analyzed for RR and QT intervals. The QT interval was corrected for heart rate using the formula $\mathrm{QT}_{\mathrm{c}}=\mathrm{QT} /(\mathrm{RR})^{1 / 2}$ using an average of ten complexes. Compared with shams $(n=8)$, experimental embryos $(n=7)$ had significantly longer $\mathrm{QT}_{\mathrm{c}}(0.339 \pm$ 0.005 versus $0.318 \pm 0.004)$, and slower heart rates $(\mathrm{RR}$ $=0.29 \pm 0.005$ versus $0.27 \pm 0.007$ ). These findings mimic those in children with the idiopathic long QT syndrome. Experimental manipulation of the sensory innervation to the heart in the chick embryo via the nodose placode may provide an animal model to improve understanding of the pathogenesis of the idiopathic long QT syndrome. (Pediatr Res 26: 11-15, 1989)
\end{abstract}

Abbreviation

$\mathrm{QT}_{c}$, heart rate corrected QT interval

The association of congenital deafness, syncope, and electrocardiographic prolongation of the QT interval has intrigued

Received September 22, 1988; accepted March 16, 1989.

Correspondence James L. Christiansen, M.D., Section of Pediatric Cardiology, University of Iowa Hospitals and Clinics, Iowa City, IA 52242.

Reprints Margaret L. Kirby, Ph.D., Heart Development Group, Department of Anatomy, Medical College of Georgia, Augusta, GA 30912-2000.

Supported by a grant-in-aid from the American Heart Association, Georgia Affiliate, and NIH Grants HL 36059 AND HD 17063. M.L.K. is an Established Investigator of the American Heart Association with funds contributed in part by the Georgia Affiliate.

This study was presented in part at the annual meeting of the Society for Pediatric Research, Washington, D.C. May 4, 1988. investigators since its first clinical description by Jervell and Lange-Nielsen in 1957 (1). Since then, a larger group of patients has been identified with inherited prolonged QT interval unaccompanied by deafness, but characterized by a dominant mode of inheritance $(2,3)$. Several pathogenetic mechanisms have been proposed to explain the idiopathic long QT syndrome and its risk of ventricular tachyarrhythmias. The most popular hypothesis supports the concept of an imbalance in the sympathetic innervation to the heart with a lower than normal right-sided cardiac sympathetic activity allowing "dispersion" of repolarization and an increased risk for ventricular arrhythmias (4). Although widely held as the cause of the long QT syndrome, the validity of this concept remains to be proven.

Recent studies on the development and interactions of cardiac innervation in the chick embryo present an opportunity to test pathogenetic mechanisms of the long QT syndrome from a developmental standpoint. The innervation of the chick heart is from at least three sets of nerves as shown in Figure $1(0,6)$. Postganglionic cardiac sympathetic nerves are found in the first thoracic sympathetic ganglia of the paravertebral sympathetic trunks. The parasympathetic postganglionic cardiac nerves (cardiac ganglia) are located on the surface of the heart. Sensory innervation to the heart arises from neurons located in the inferior or thoracic ganglia of the vagus (comparable to the nodose ganglion in mammals). The neuronal precursors of sensory cardiac innervation are derived from the nodose placode. The parasympathetic and sympathetic innervation of the heart are derived from neural crest. Parasympathetic cardiac ganglia arise from neural crest adjacent to somites 1-3. Sympathetic postganglionic neurons arise from the neural crest adjacent to somites 10-20. The nodose placode is an ectodermal thickening on the dorsal embryonic surface caudal but adjacent to the otic placode from which the inner ear develops.

As one phase of a study to assess the effects of alterations of cardiac innervation on electrocardiographic activity, we sought to determine whether selective perturbation of sensory innervation to the developing chick heart could alter the QT interval of the embryonic chick ECG.

\section{MATERIALS AND METHODS}

Fertile Arbor Acre chicken eggs (Seaboard Farms, Athens, GA) were obtained fresh and stored at $15-20^{\circ} \mathrm{C}$ until incubation. Eggs were incubated in a forced-draft incubator maintained at $38^{\circ} \mathrm{C}$ and $97 \%$ humidity for $32-35 \mathrm{~h}$. The eggs were opened at Hamburger-Hamilton (7) stage 10 of development using a disc sander and prepared for microsurgery according to the method reported by Narayanan (8). Embryonic structures were made visible by lightly staining with the vital dye, neutral red. The vitelline 


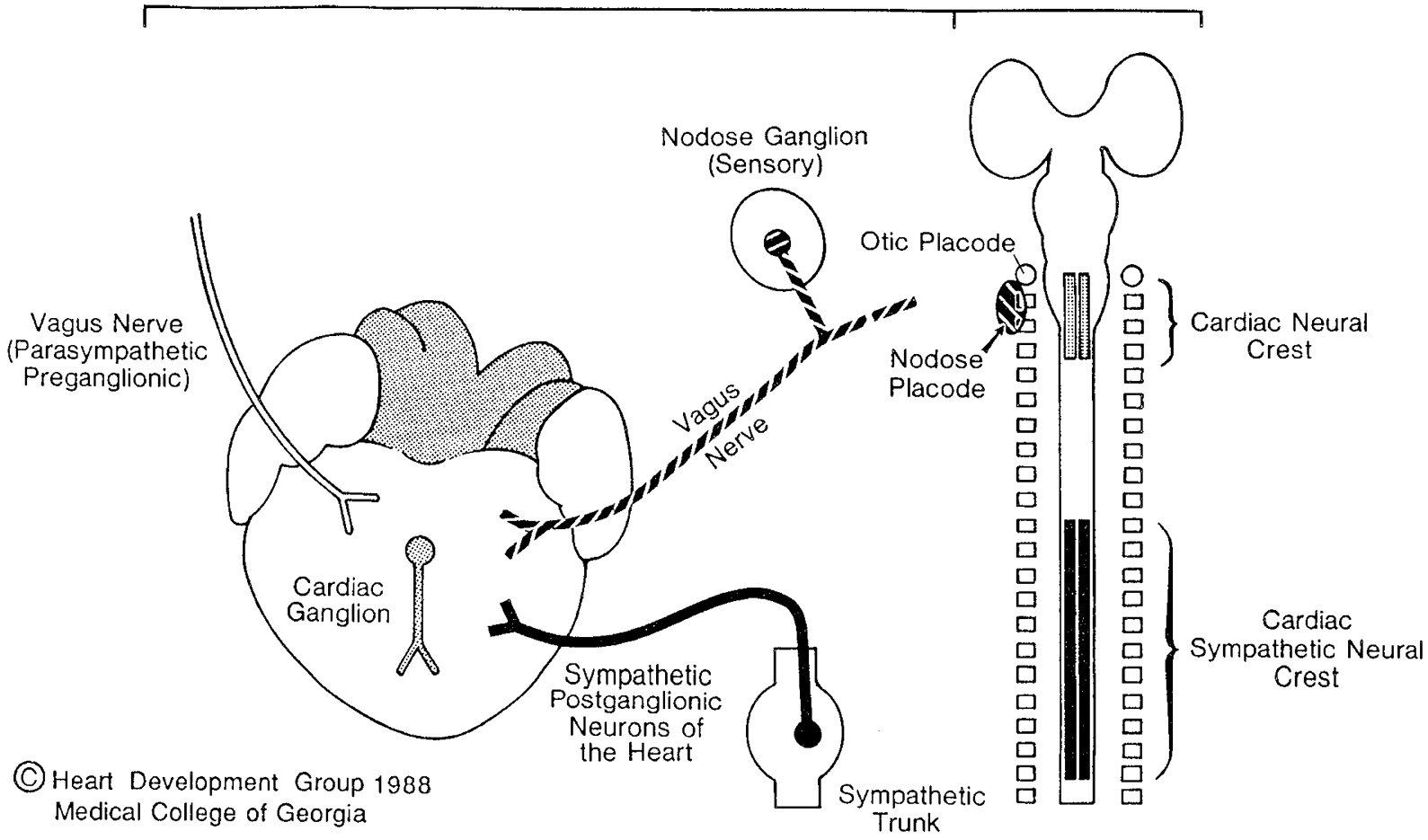

Fig. 1. Illustration of the relationship of cardiac innervation and its derivation. The parasympathetic postganglionic neurons are derived from neural crest adjacent to somites 1-3 (cardiac neural crest) (gray shading). The cardiac sympathetic postganglionic neurons (sympathetic trunk) arise from trunk neural crest adjacent to somites 10-20 (highlighted in black). The sensory innervation of the heart is derived from cells which arise from the nodose placode, forming neurons in the inferior ganglion of the vagus (nodose ganglion). Note the proximity of the nodose placode to the otic placode, from which are derived structures of the inner ear.

\begin{tabular}{|c|cc|}
\hline Switch & $\begin{array}{c}\text { Recording between } \\
\text { electrodes }\end{array}$ \\
1 & $1+\mathrm{II}$ \\
2 & $1+\mathrm{III}$ \\
3 & $\mathrm{II}+\mathrm{III}$ \\
\hline
\end{tabular}

Fig. 2. Set-up for recording electrocardiograms in the chick embryo. Leads were connected to a switching box to allow sequential recording.

membrane was then torn to expose the embryonic structures of interest.

Microsurgery. Premigratory surface ectoderm in the area of the right nodose placode (Fig. 1) was removed using a microcautery needle (Biomedical Engineering Department, Medical College of Georgia). A unilateral lesion was chosen in part to limit mortality associated with surgical manipulation. Eggs were then sealed with cellophane tape and returned to the $38^{\circ} \mathrm{C}$ incubator. When the circulation was established (about $72 \mathrm{~h}$ of incubation) eggs were moved to an incubator maintained at $37^{\circ} \mathrm{C}$ and $70 \%$ humidity until ready for use. Sham-operated controls were prepared in parallel, undergoing identical preparatory procedures without removal of the nodose placode.

Electrocardiography. At incubation days 17-20, three lead ECG were obtained in an electrically grounded, controlled temperature environment. Four $0.5-\mathrm{mm}$ stainless steel electrodes were inserted through the shell as depicted in Figure 2. Lead orientation was kept constant to allow comparison of results between embryos. Bipolar electrograms were obtained using lead pairs and a grounded reference electrode. A thermistor was placed within the egg to monitor temperature which was kept constant at $37 \pm 0.2^{\circ} \mathrm{C}$. Electrocardiographic leads were connected to a switching box to allow sequential recording of lead pairs. This in turn was connected with an isolated preamplifier (Gould Electronics, Norcross, GA) and amplifier (Gould Universal) with a frequency response of 0.05 to $100 \mathrm{~Hz}$. A chart recorder (Gould RS 3200) was used to record electrocardiograms at 50 and $100 \mathrm{~mm} / \mathrm{s}$ paper speeds. The best quality lead tracing was used to measure the R-R and Q-T intervals. The Q-T interval was then corrected for heart rate using the formula $\mathrm{QT}_{\mathrm{c}}=\mathrm{QT} /$ $(\mathrm{RR})^{1 / 2}$ and averaged for 10 complexes. Measurement of intervals (to the nearest $5 \mathrm{~ms}$ ) was accomplished using hand-held calipers. The ECG were analyzed without knowledge of embryo grouping. Embryos were excluded from analysis if high-quality electrocardiograms could not be obtained. Representative electrocardiograms are shown in Figure 3.

Pathologic analysis. After electrocardiography, embryos were killed, analyzed for gross defects, and staged for maturity using the criteria published by Hamburger and Hamilton (7). The heart was rapidly perfused with Carnoy's fixative and the embryo was postfixed by immersion in $10 \%$ neutral buffered formalin. The heart and great vessels were examined grossly for abnormalities, then detailed dissection was undertaken to delineate the course and nature of the aortic arch and its branches, as well as to catalogue intracardiac defects, if any. Additional note was made of the presence and size of the nodose ganglia (inferior ganglia of the vagus). Embryos with severe gross defects were excluded from analysis.

Statistical methods. Results of electrocardiographic analysis for $\mathrm{RR}$ and $\mathrm{QT}_{\mathrm{c}}$ for sham and experimental groups were compared using ANOVA and the unpaired one-tailed $t$ test. A $p$ value of $<0.05$ was considered to reflect a significant difference between the two groups. 


\section{RESULTS}

A total of 114 embryos was subjected to microcautery or sham operation (84 right nodose placode ablation, 30 sham operation). Viability ranged from $24 \%$ in the experimental group to $40 \%$ in the sham-operated controls. These rates compared favorably with viability rates in previous studies using the same techniques $(9$,

\section{SHAM}
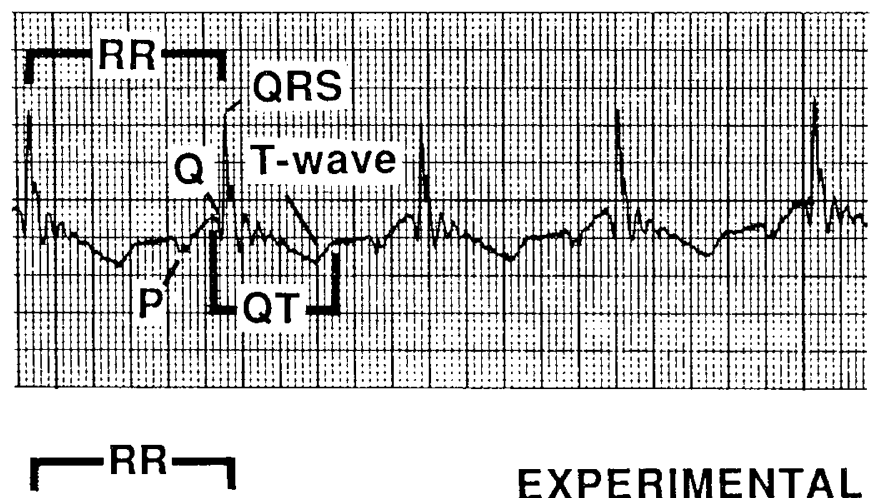

EXPERIMENTAL
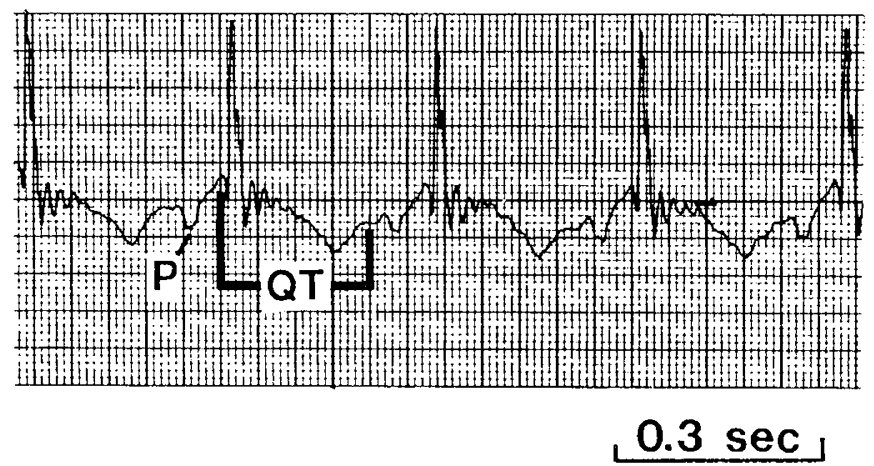

Fig. 3. Representative electrocardiograms recorded at $100 \mathrm{~mm} / \mathrm{s} \mathrm{pa-}$ per speed from sham-operated and experimental embryos. The QT interval was corrected for heart rate using the formula $\mathrm{QT}_{\mathrm{c}}=\mathrm{QT} /(\mathrm{RR})^{1 / 2}$. The QTc for the sham-operated embryo was calculated to be 0.299 whereas in the experimental embryo the value was 0.354 .
10). There were 32 embryos that survived for study. A total of 17 embryos was excluded from analysis, 12 because of poor quality electrocardiographic tracings, five because of severe malformations.

Fifteen embyos were selected for analysis as shown in Table 1. Embryos subjected to right nodose placode ablation had longer rate corrected QT intervals than sham-operated embryos ( $p=$ 0.008 ). Further comparison of the experimental group to a larger reference population of embryos not subjected to microsurgery yielded similar results $(p<0.001)$ (Table 2$)$. Additionally, experimental embryos had lower average heart rates than shamoperated controls $(p=0.02)$, mean 207 beats/min versus 222 beats/min. No arrhythmias were detected during electrocardiographic monitoring. Gross and pathologic analysis of both groups is depicted in Table 3. Embryos from both groups were affected. Three embryos in the experimental group and two in the sham-operated control group had cardiac or other structural defects. Cardiac defects were confined to the area of the membranous interventricular septum where a small ventricular septal defect was present in four embryos. Analysis of the outflow region and aortic arch vessels of these embryos revealed no abnormalities. The right and left nodose ganglia were located in all embryos. A qualitative size comparison between the two showed mild hypoplasia of the right nodose ganglion (ipsilateral to microcautery ablation) in the three embryos with more

Table 1. Results of electrocardiogram analysis (mean \pm SEM)

\begin{tabular}{llcc}
\hline \multicolumn{1}{c}{ Group } & $n$ & $\mathrm{RR}$ & $\mathrm{QT}_{c}$ \\
\hline Experimental & 7 & $.29 \pm .005$ & $.339 \pm .005$ \\
Sham & 8 & $.27 \pm .007$ & $.318 \pm .004$ \\
& & $\mathrm{p}=.02$ & $\mathrm{p}=.008$ \\
\hline
\end{tabular}

Table 2. Distribution of heart rate corrected $Q T$ interval related to age in embryos not subjected to nodose placode ablation (mean $\pm S E M)$

\begin{tabular}{crc}
\hline Embryo age (day) & $n$ & QT $_{c}$ \\
\hline 17 & 9 & $0.304 \pm 0.004$ \\
18 & 16 & $0.307 \pm 0.005$ \\
19 & 9 & $0.304 \pm 0.006$ \\
20 & 10 & $0.308 \pm 0.004$ \\
\hline
\end{tabular}

Table 3. Pathologic analysis of embryos*

\begin{tabular}{|c|c|c|c|c|}
\hline & \multicolumn{3}{|c|}{ Observed defects } & \multirow[b]{2}{*}{$\mathrm{QT}_{c}$} \\
\hline & Heart & Nodose ganglion & Other & \\
\hline \multicolumn{5}{|c|}{ Experimental animal } \\
\hline 1 & VSD & Normal & Hypoplastic maxilla encephalocele & 0.329 \\
\hline 2 & Normal & Normal & None & 0.324 \\
\hline 3 & VSD & Normal & Mild abdominal wall defect & 0.338 \\
\hline 4 & Normal & Normal & None & 0.334 \\
\hline 5 & Normal & $\mathrm{R}$ hypoplastic & None & 0.339 \\
\hline 6 & Normal & $\mathrm{R}$ hypoplastic & $\begin{array}{l}\text { Mild abdominal wall defect } \\
\text { Mild ectopia cordis }\end{array}$ & 0.350 \\
\hline 7 & Normal & R hypoplastic & None & 0.359 \\
\hline \multicolumn{5}{|c|}{ Sham-operated animal } \\
\hline 1 & Normal & Normal & None & 0.317 \\
\hline 2 & Normal & Normal & None & 0.296 \\
\hline 3 & Normal & Normal & None & 0.321 \\
\hline 4 & VSD & Normal & None & 0.309 \\
\hline 5 & Normal & Normal & None & 0.301 \\
\hline 6 & Normal & Normal & None & 0.330 \\
\hline 7 & Normal & Normal & None & 0.333 \\
\hline 8 & VSD & Normal & Encephalocele & 0.333 \\
\hline
\end{tabular}

* VSD, ventricular septal defect; $R$, right. 
marked QT prolongation. Extracardiac defects were more frequently seen in the experimental group, but rate corrected QT prolongation did not appear to be related to the presence of extracardiac defects.

\section{DISCUSSION}

Prolongation of the QT interval on the surface ECG is representative of prolonged or delayed ventricular repolarization (11). Although acquired conditions such as metabolic and electrolyte disturbances, hypothermia, drug therapy, and cardiac trauma predominate in the classification scheme, the idiopathic or congenital types with or without heritable features remain the major focus of interest (11). In a recent review, Schwartz (4) discussed the pathogenetic mechanisms proposed for these latter categories. The strong association between syncopal episodes and physical or emotional stress implied a critical role of the sympathetic nervous system in the genesis of arrhythmias typical of the syndrome. The basic premise that the increased risk of ventricular arrhythmias was a consequence of an imbalance in the sympathetic innervation to the heart, with an underactivity of the right-sided sympathetic cardiac nerves, was supported by circumstantial evidence from both human and animal studies (12-15). Findings that implicated an abnormality of right-sided sympathetic cardiac nerves included data showing that: 1) interruption of the right cardiac sympathetic activity in dogs mimics the characteristic electrocardiographic changes, 2) sympathetic control of heart rate is almost exclusively mediated through rightsided cardiac nerves with most affected patients manifesting a low resting heart rate and impaired chronotropic response to exercise, and 3) right stellate ganglion destruction in dogs lowers the threshold for ventricular fibrillation. Although various acute animal preparations have been proposed as models because they have symptoms mimicking those of the long QT syndrome, there is as yet no suitable animal model from which to study developmentally, the mechanisms which may underlie the evolution of these conditions.

The interactions of parasympathetic, sympathetic, and sensory nerves that result in an appropriate balance of neural input to the developing heart are poorly understood. Recent studies in the chick and mouse embryo have advanced knowledge of these relationships. The production of sympathetically aneural chick hearts by removing neural crest cell precursors of sympathetic innervation, has enabled study of the effects of removing only one division of the autonomic input to the heart $(16,17)$. When the sympathetic innervation to the developing heart was removed, parasympathetic innervation was increased by $50-100 \%$ due to both hypertrophy and hyperplasia of the ganglion cells as well as their terminals (18). Studies in the adult mouse iris have further demonstrated the reciprocal modulation of sensory, sympathetic, and parasympathetic terminals in the target organ (19, 20). Removal of any one set of nerves allowed reciprocal increases in growth of the remaining nerves, possibly by altering the availability of growth factors produced by the target organ.

The chick embryo is an easily manipulated animal in which to study interactions of the autonomic nervous system as they might relate to pathogenesis of the idiopathic long QT syndrome. As compared to murine systems, the development and functional activity of autonomic innervation to the heart in avians occurs early $(21,22)$. This feature allows study of possible perturbations of neural input to the heart in a functionally intact system before hatching. It has been established that cholinergic neuroeffector transmission begins on day 12 of incubation, whereas functional adrenergic transmission is not demonstrable until embryonic day 16 (6). Therefore, our characterization of electrocardiographic changes at embryonic day 17 or later has the potential for extrapolation to possible autonomic interactions later in life as the sympathetic and parasympathetic nervous systems mature.

We present data to show that perturbation of the anlage of cells destined to provide sensory innervation to the heart, prior to their migration, results in prolongation of the QT interval of the embryonic chick electrocardiogram. Additionally, the mean heart rate of affected embryos was lower than that of shamoperated controls. These findings mimic those of children with the idiopathic form of the long QT syndrome (23). The mechanism of these changes is not known. Whether they represent a direct result of deficient sensory innervation, or are secondary to an induced imbalance of sympathetic or parasympathetic innervation, remains to be determined by future studies. The presence of mild hypoplasia of the right nodose ganglion in several embryos with long $\mathrm{QT}_{\mathrm{c}}$ implicates some residual effect of nodose placode ablation early in development. Although hypoplastic, the right nodose ganglion remained present which is consistent with knowledge of the contribution of other cell precursors to its makeup, namely neural crest (5). The nodose placode contributes neurons to the nodose ganglion, although the supporting elements and satellite cells are supplied by neural crest. An alternative hypothesis is that total ablation of all nodose placode cells is lethal at this embryonic age, even if performed in a unilateral fashion. This may explain the high mortality associated with this lesion. Additionally, as expected, all animals did not show the same degree of QT prolongation or change in heart rate. Nodose placode microcautery was difficult to perform in a reproducible fashion, thus allowing for a variable effect on QT interval depending upon the number of placodal cells destroyed or that possibly regenerated.

In our opinion, the occurrence of heart and other structural defects in these embryos does not weaken the utility of this model. Spontaneous occurrence of ventricular septal defects has been known to occur in White Leghorn chick embryos (24). The lack of associated aortic arch anomalies in the experimental group is an important finding to exclude an additional effect of microcautery on cardiac neural crest. Cardiac neural crest, in addition to supplying mesenchymal elements to the aorticopulmonary septum and aortic arches, supplies all of the postganglionic parasympathetic innervation to the heart $(6,25)$. Similarly, the more frequent occurrence of midline and chest wall defects in the experimental groups may reflect the extensive manipulation required to successfully perform microcautery. In addition, the first surgical procedure, tearing the vitelline membrane, performed on embryos in both experimental and control groups, may cause significant lateral tension resulting in partial separation of the neural tube. This may explain the occasional occurrence of encephalocele in each group. Finally, the finding of maxillary hypoplasia (upper beak shorter than lower beak) in one experimental animal may imply some effect on migrating cranial neural crest, as these cells form the skeleton of the upper and lower jaws in addition to other skeletal structures in the head (26). Although cephalic to the nodose placode, neural crest cells in this region may have been inadvertently damaged by manipulation or microsurgical procedures.

Our use of Bazett's (27) formula for the correction of QT interval for variation in heart rate may be an oversimplification of the relationship of QT and heart rate for the developing chick embryo. To our knowledge such a relationship for chick embryos has never been studied and would be difficult given the narrow range of heart rate that characterizes this stage of embryonic development. Lead orientation was standardized as far as possible given the possibility that changes in lead position might affect the QT duration $(28,29)$. Lead positioning by direct visualization and impalement of the embryo was deemed unsatifactory due to inability to control for resulting neural stimulation that may have varied widely among embryos. We believe that the possible contribution of the effect of lead orientation to QT interval measurement was more than compensated for by measurement of tracings at $100 \mathrm{~mm} / \mathrm{s}$ and selection of the best quality tracing for definition of $\mathrm{T}$ wave intersection with isoelectric baseline. Finally, although we eliminated obvious acquired causes for QT prolongation, e.g. hypothermia and exogenous drugs, we did not evaluate other possibilities such as electrolyte or metabolic dis- 
turbances. It seems very remote that these conditions would be operant at the time of analysis when the initial perturbation occurred so early in development.

The value of this model as currently developed lies in its potential to provide new insights into the autonomic interactions that may be the basis for the idiopathic long QT syndrome. Our speculation that this syndrome may be related to perturbations of sensory innervation to the heart is important in that the contiguity of the otic and nodose placodes early in embryonic development, in man as well as avians, provides a developmental link to the poorly understood association of hearing abnormalities that characterize one presentation of the syndrome (30) (See Fig. 1). Injury to this area early in development has the potential to cause hearing loss with perturbation of cardiac innervation.

Acknowledgments. The authors wish to thank Karen Waldo for the graphic arts, Dr. Harry Davis for statistical assistance, and Susan Dunham for secretarial assistance.

\section{REFERENCES}

1. Jervell A, Lange-Nielsen F 1957 Congenital deaf-mutism, functional heart disease with prolongation of the Q-T interval and sudden death. Am Heart J 54:59-68

2. Romano C, Gemme G, Pongiglione R 1963 Aritmie cardiache rare dell'eta pediatrica. Clin Paediatr 45:656-683

3. Ward OC 1964 A new famial cardiac syndrome in children. J Irish Med Assoc 54:103-106

4. Schwartz PJ 1985 Idiopathic long QT syndrome: Progress and questions. Am Heart J 109:399-411

5. d'Amico-Martel A, Noden DM 1983 Contributions of placodal and neura crest cells to avian cranial peripheral ganglia. Am J Anat 166:445-468

6. Kirby ML, Stewart DE 1986 Development of the ANS innervation to the avian heart. In: Gootman P (ed) Developmental Neurobiology of the Autonomic Nervous System. Humana Press, Clifton, NJ, pp 135-158

7. Hamburger V, Hamilton HV 1951 A series of normal stages in the development of the chick embryo. J Morphol 88:49-62

8. Narayanan $\mathrm{CH} 1970$ Apparatus and current techniques in the preparation of avian embryos for microsurgery and for observing embryonic behavior. Bioscience 20:868-871

9. Kirby ML, Aronstam RS, Buccafusco JJ 1985 Changes in cholinergic parameters associated with failure of conotruncal septation in embryonic chick hearts after neural crest ablation. Circ Res 56:392-401

10. Nishibatake M, Kirby ML, Van Mierop LHS 1987 Pathogenesis of persistent truncus arteriosus and dextroposed aorta in the chick embryo after neura crest ablation. Circulation 75:255-264
11. Surawicz B, Knoebel SB 1984 Long QT: good, bad, or indifferent? J Am Coll Cardiol 4:398-413

12. Yanowitz R, Preston JB, Abildskov JA 1966 Functional distribution of right and left stellate innervation to the ventricles: production of neurogenic electrocardiographic changes by unilateral alternation of sympathetic tone. Circ Res 18:416-428

13. Randall WC, Rohse WG 1956 The augmentor action of the sympathetic cardiac nerves. Circ Res 4:470-475

14. Schwartz PJ, Stone HL 1976 Role of right stellate ganglion during exercise. Eur J Clin Invest 6:328(abstr)

15. Schwartz PI Snebold NG, Brown AM 1976 Effects of unilateral cardiac sympathetic denervation on the ventricular fibrillation threshold. Am J Cardiol 37:1034-1040

16. Kirby ML, Stewart DE 1984 Adrenergic innervation of the developing chick heart. Neural crest ablations to produce sympathetically aneural hearts. Am J Anat 171:295-305

17. Phillips MT, Kirby ML, Stewart DE 1986 Cyclic AMP in normal and sympathetically aneural hearts during development. J Mol Cell Cardiol 18:827835

18. Kirby ML, Conrad DC, Stewart DE 1987 Increase in the cholinergic cardiac plexus in sympathetically aneural chick hearts. Cell Tissue Res 247:489-496

19. Kessler JA 1985 Parasympathetic, sympathetic, and sensory interactions in the iris: nerve growth factor regulates cholinergic ciliary ganglion innervation in vivo. J Neurosci 5:2719-2725

20. Kessler JA, Bell WO, Black IB 1983 Interactions between sympathetic and sensory innervation of the iris. J Neurosci 3:1301-1307

21. Pappano A 1975 Development of autonomic neuroeffector transmission in the chick embryo heart. In Lieberman M, Sano $T$ (eds) Developmental and Physiological Correlates of Cardiac Muscle. Raven Press, New York, pp 235-247

22. Marvin WJ, Hermsmeyer K, McDonald RI, Roskoski LM, Roskoski R 1980 Ontogenesis of cholinergic innervation in the rat heart. Circ Res 46:690-695

23. Vincent GM 1986 The heart rate of Romano-Ward syndrome patients. Am Heart J 112:61-64

24. Kuhlmann RS, Kolesari GL 1984 The spontaneous occurrence of aortic arch and cardiac malformations in the White Leghorn chick embryo (Gallus domesticus). Teratolgy 30:55-59

25. Kirby ML 1987 Cardiac morphogenesis-recent research advances. Pediat Res 21:219-224

26. Le Douarin N 1982 The Neural Crest. Cambridge University Press, Cambridge, pp 54-90

27. Bazett HC 1920 An analysis of the time-relations of electrocardiograms. Heart $7: 353-370$

28. Sylven JC, Horacek BM, Spencer CA, Klassen GA, Montague TJ 1984 QT interval variability on the body surface. J Electrocardiol 17:179-188

29. Cowan IC, Yusoff K, Moore M, Amos PA, Gold AE, Bourke JP, Tansuphaswadikul S, Campbell RWF 1988 Importance of lead selection in QT interval measurement. Am J Cardiol 61:83-87

30. Kissel P, Andre JM, Jacquier A 1981 The Neurocristopathies. Masson Publishing, New York, pp 1-14 